\title{
BMJ Open Quality Reducing urinary tract infections in care homes by improving hydration
}

\author{
Katie Lean, ${ }^{1}$ Rasanat Fatima Nawaz, ${ }^{1,2}$ Sundus Jawad, ${ }^{3}$ Charles Vincent ${ }^{2}$
}

To cite: Lean K, Nawaz RF, Jawad S, et al. Reducing urinary tract infections in care homes by improving hydration. BMJ Open Quality 2019;8:e000563. doi:10.1136/ bmjoq-2018-000563

Received 25 0ctober 2018 Revised 21 May 2019 Accepted 24 June 2019
Check for updates

(C) Author(s) (or their employer(s)) 2019. Re-use permitted under CC BY-NC. No commercial re-use. See rights and permissions. Published by BMJ.

${ }^{1}$ Patient Safety, Oxford Patient Safety Collaborative, Oxford, UK ${ }^{2}$ Department of Experimental Psychology, University of Oxford, Oxford, UK

${ }^{3}$ Medicines Optimisation Team, NHS East Berkshire Clinical Commissioning Group, Windsor, UK

Correspondence to

Katie Lean;

katie.lean@oxfordahsn.org

\begin{abstract}
Dehydration may increase the risk of urinary tract infections (UTIS), which can lead to confusion, falls, acute kidney injury and hospital admission. We aimed to reduce the number of UTIs in care home residents which require admission to hospital. The principal intervention was the introduction of seven structured drink rounds every day accompanied by staff training and raising awareness. UTIs requiring antibiotics reduced by $58 \%$ and UTIs requiring hospital admissions reduced by $36 \%$, when averaged across the four care homes. Care home residents benefited from greater fluid intake, which in turn may have reduced infection. Structured drink rounds were a low-cost intervention for preventing UTIs and implemented easily by care staff.
\end{abstract}

\section{PROBLEM}

Urinary tract infection (UTI) was the condition with the highest rate of emergency admissions to hospital in 2012/2013. ${ }^{1}$ Dehydration has been highlighted as a common cause of admission to hospital in nursing home residents, ${ }^{2}$ and there is evidence that many older residents living in care homes do not receive enough fluids. In this context, a care home is a residential environment for older adults providing them with onsite care services where required.

The regional acute kidney injury programme at the Oxford Patient Safety Collaborative was established to improve the recognition and response to acute kidney injury. East Berkshire Clinical Commissioning Group (CCG) highlighted that UTI was the most common reason for hospital admission from their care home population in $2015 / 2016$. A quality improvement project was designed to address the aim of improving hydration and reducing UTIs.

East Berkshire CCG has over 75 care homes within their region. We identified four care homes with the highest incidence of UTI admissions to hospital and approached them to collaborate and co-design the project. These three residential homes and one nursing home had a combined total of 150 residents, majority over 75 years old and more than half living with dementia. Most of the employees are care assistants who work 12-hour shifts and in one home there was a high turnover of staff. All homes remained in the project from conception to completion.

The SMART aim of this quality improvement project was to reduce the incidence of UTIs by improving hydration with the ultimate aim of reducing UTI admissions to hospital by $5 \%$ from the previous year.

\section{BACKGROUND}

Promoting good hydration and nutrition in older adults leads to increased well-being and improved quality of life. ${ }^{3}$ Older adults often forget to drink ${ }^{4}$ and over half of nursing home residents do not have a safe swallowing mechanism making them susceptible to decreased fluid intake. ${ }^{5}$ These factors increase the risk of dehydration and UTIs in older adults, which can in turn lead to confusion, falls, acute kidney injury and hospital admission. ${ }^{6}$ Inadequate staffing, including high turnover and understaffed care homes, increases the risk of dehydration in residents. ${ }^{78}$

Dysphagia (difficulty in swallowing) is also known to contribute to dehydration in older adults. Over half of care home residents are highlighted as having issues swallowing. ${ }^{5} 9$ Older adults with dysphagia will require more encouragement to drink as fluids become less palatable and require thickeners. There is also an increased fear of choking, drooling and aspirating in residents. ${ }^{10}$ Residents with dementia may require 1:1 care as they are at a higher risk of dehydration and require careful support and management with their drinking and eating. ${ }^{11}$

Residents with a safe swallow have an increased risk of dehydration due to the decline of the sense of thirst in older adults. ${ }^{12}$ Many nursing home residents drink less than 1.5 litres a day and leave drinks unfinished. ${ }^{12} 13$ Older adults capable of drinking without assistance may also limit fluid intake due to concerns surrounding incontinence as well as the fear of falling when needing to go to the toilet. ${ }^{14}$

Monitoring fluid intake of patients and residents is common in hospital and care 
environments. ${ }^{4}$ Fluid balance charts are used to record fluid intake and output. Drinks intake charts are used to exclusively record intake of drinks. Ensuring accurate documentation has been a challenge when using these methods. Numerous studies have identified inaccuracies across the use of these charts, particularly in the residential care setting. ${ }^{15-17}$ In one study, ${ }^{15}$ a third of residents were at risk of dehydration but not identified as such through their fluid intake charts. Accurate fluid charts are critical to improving hydration, but their design and use may need to be adapted to suit the care home environment.

In a small study, drinks diaries were completed by patients to track fluid intake over a 24-hour period. ${ }^{18}$ Residents were typically asked to record the name of the drink, type of mug, cup or glass used, and tick the appropriate picture indicating how much they drank (a little, half, a lot or all). Due to cognitive or physical disabilities, many residents were unable to keep their own drinks diaries. However, the intervention led to increased fluid intake for those residents who had the ability and capacity to write. ${ }^{18}$

A small number of studies have attempted to improve hydration in nursing homes. For example, a 5-week hydration programme was carried out in the USA, with a sample of 51 nursing home residents aiming to increase daily fluid intake by 16 ounces. ${ }^{19}$ The intervention involved increasing the presentation of drinks using a more attractive format, for example, decorated drinks carts. Over half the residents achieved the target additional 16 fluid ounces. The study did not find a statistically significant improvement in UTI reduction; however, other researchers have cited poorer physical health and UTI as one of the conditions that older adults are susceptible to when they do not receive adequate hydration. ${ }^{20}$

In summary, there is evidence that improving hydration has a variety of health benefits for older residents living in care homes, but there are few validated interventions. Structured drink rounds appear to be the most promising approach, but there is little guidance on how best to implement these. The intervention should allow for care home staff to receive relevant training in the importance of hydration, while offering and monitoring fluids in a flexible manner that suits each care home.

\section{MEASUREMENT}

The project started in May 2016 when formal baseline data were collected for the number of UTIs requiring antibiotics. Hospital admissions data were accessed from May 2015 and monitored throughout the project. The underlying population is the same in the study as the data were collected from four care homes and the same overall number of residents. Naturally, there is some change over time in the residents in each home.

Baseline data for UTI admission to hospital were provided by Secondary Uses Service accessed by East Berkshire CCG. The data measured all residents who were admitted to hospital with a UTI as the primary diagnosis. Baseline data were collected from May 2015 to June 2016, across the four care homes and collected monthly through the CCG from July 2016 to March 2018.

Baseline data for the number of UTIs requiring antibiotic treatment each month were collected using Safety Crosses, between May 2016 and June 2016. A safety cross is a visual tool containing 31 boxes (each box counts as a day in the month) used to collect data for improvement. A nominated champion within the care home was responsible for completing these daily. A red sticker indicated if a resident had a UTI requiring admission to hospital, an orange sticker indicated a resident requiring an antibiotic prescription for a UTI and a green sticker was an incident-free day. A sticker was placed on the date the UTI occurred, and if multiple residents had a UTI on the same day, multiple stickers would be used. During the project, the antibiotics data were collected monthly from July 2016 to March 2018.

Structured drink rounds were implemented seven times a day as the principal intervention and recorded on a designated sheet by the carer undertaking each round. The project champion would then tally the daily totals to give a weekly percentage of completed drink rounds. These data were then entered into a run chart by the project lead to note variation, sustainability of the process and offer support when required. Staff collated this information at the end of each month, allowing them to highlight successes and also identify areas for improvement.

\section{DESIGN}

The SMART aim of this quality improvement project was to reduce the incidence of UTIs by improving hydration with the ultimate aim of reducing admissions to hospital by $5 \%$ from the previous year.

All aspects of the project and intervention were designed and implemented in close collaboration with the care homes. A multidisciplinary team was formed to oversee the project which included a patient safety manager, pharmacist, dietitian, local general practitioner (GP), care home staff including managers, nurses, carers, activity co-ordinators and chefs. Throughout the project, care home residents were approached for their thoughts on the drink rounds.

Training of staff on the signs and symptoms of UTIs was identified as the first priority. This ensured SIGN 88 guidance $^{21}$ was used to identify clinical signs and symptoms of a UTI rather than the use of routine dipstick testing of residents. Hydration champions were identified to cascade the information and posters designed as this was more feasible than training busy staff individually.

The principal intervention was the implementation of a structured drink round to ensure that residents were offered a wide selection of hot and cold drinks at least seven times a day. 


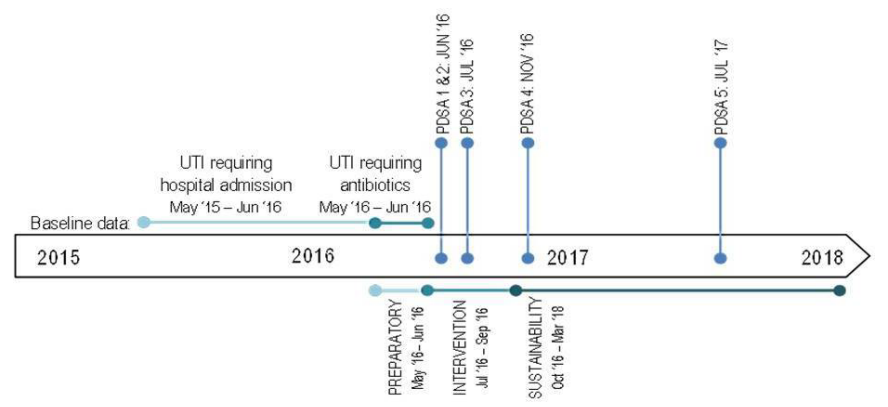

Figure 1 Timeline of the project illustrating when baseline data were collected, implementation of Plan, Do, Study, Act (PDSA) cycles and different phases of the project; preparatory, intervention and sustainability. UTI = urinary tract infection.

\section{STRATEGY}

The principal intervention was the introduction of seven structured drink rounds. This was supported by staff training, awareness and campaigns, drinks diaries for residents at risk of dehydration and guidance for sustaining changes in the long term (see figure 1). Plan, Do, Study, Act (PDSA) cycles were used to trial the components of the improvement intervention implemented to achieve these aims.

\section{Preparatory phase}

PDSA cycle 1: A poster was designed to raise awareness of the signs and symptoms of a UTI. The poster displayed information on the definition and diagnosis of a UTI as per SIGN 88 guidance. ${ }^{21}$

PDSA cycle 2: A 2-hour training session was attended by care home managers, nurses, support workers, activities co-ordinators and chefs. The training was open to all staff within the care home to ensure the importance of hydration was understood across all teams. The sessions covered the anatomy and physiology of the urinary system, signs and symptoms of dehydration, causes of dehydration, methods to improve hydration, effects of certain medication on kidneys and signs and symptoms of a UTI. At the end of the training sessions staff discussed the importance of structured drink rounds and ideas and thoughts on how to adapt these to suit their care home.

\section{Intervention phase}

PDSA cycle 3: The seven daily structured drink rounds were designed by the care homes to encourage hydration at regular intervals throughout the day for all residents. Seven drink rounds were chosen due to it being manageable for care home staff and supporting the daily recommendation of $6-8$ glasses of water to keep residents hydrated. ${ }^{22}$ The care homes decided what times they were going to undertake the drink rounds.

The drink round included theming and decorating the trolleys, ensuring they were bright and appealing for residents and to attract the attention of those with dementia. The theme was changed regularly to stimulate residents and keep up with key events, for example, festivals or sporting events. A wide variety of hot and cold drinks were presented using colourful juices, colourful cups and mugs. Ice lollies and milkshakes were included to offer alternative methods of increasing fluid intake. Residents were consulted throughout the process and selected cocktails of the month and taste tested various drinks to decide what they preferred.

Staff recorded drink rounds on a designated sheet and were required to circle 'yes' or 'no' depending on whether the drink round was carried out or not. They were encouraged to be honest about whether the drink round was undertaken. If any rounds were not taking place, then this allowed staff to review the times at which the drinks were being offered or other confounding issues.

\section{Sustainability phase}

PDSA cycle 4: Supplementary drinks diaries were introduced in the nursing home for all residents, and in the remaining three residential homes, they were introduced on an ad hoc basis whenever there were concerns about a risk of dehydration. Residents with capacity completed these themselves, but most of the time staff completed them. The diaries were designed, trialled and adjusted to suit the needs of the residents, staff and home. They documented the drinking behaviour of each resident, measured the type of drink and recorded the quantity consumed.

PDSA cycle 5: Good Practice Guidance (GPG) was written to encourage care home staff to understand clinical signs and symptoms of a UTI as per SIGN 88 guidance, ${ }^{21}$ as well as promoting good hydration. A form to communicate UTI signs and symptoms was developed for care staff to use when communicating with GPs but also serves as a UTI management care plan.

GPG encouraged GPs to move away from routinely requesting a urine dipstick test in residents over 65 years old as a confirmation for diagnosis and recommended local formulary antibiotic prescribing information and guidance. The guidance was approved by East Berkshire Clinical Commissioning Group Effective Prescribing Performance Committee and introduced to assist care home staff and GPs to work collaboratively in effectively identifying UTIs and optimising treatment and management.

\section{RESULTS}

\section{UTIs requiring antibiotics}

The average number of UTIs requiring antibiotics across the four care homes at baseline was 1.8, and this reduced to 0.75 UTIs during the intervention and sustainability phase (see figure 2). This resulted in a $58 \%$ reduction of UTIs requiring antibiotics post intervention (MannWhitney $\mathrm{U}=6.00, \mathrm{p}=0.10)$.

During the baseline period, the average number of days between UTIs was 9 days. As the intervention was put in place, this increased to 44 days. After 12 months of the intervention, the days between UTIs increased to 121 and 
UTIs requiring antibiotics

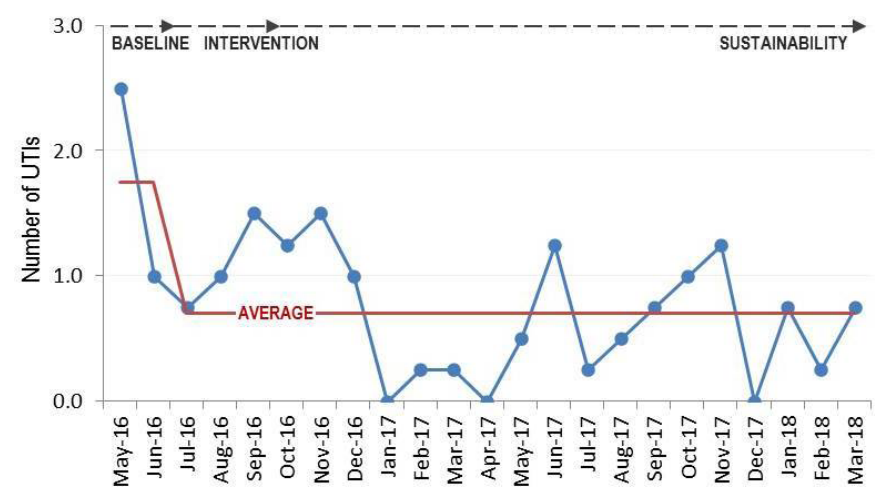

Figure 2 Average monthly numbers of urinary tract infections (UTIs) requiring antibiotics during the baseline, intervention and sustainability phase.

at 18 months this was 80 days without UTIs across all four care homes.

\section{Hospital admissions}

Across the four care homes, the average number of UTIs requiring hospital admission at baseline was 1.4 and reduced to 0.9 UTIs during the intervention and sustainability phase (see figure 3 ). This resulted in a $36 \%$ reduction of UTIs requiring hospital admission (Mann-Whitney $\mathrm{U}=83.00, \mathrm{p}=0.09$ ).

\section{Sustainability}

Across the four care homes, staff maintained a monthly drink rounds compliance of more than $97 \%$. Compliance increased from $97 \%$ during the intervention phase to consistently over $99 \%$ in the sustainability phase.

One care home noted that they were always missing a drinks round at 13:30 and felt that it was too close to lunch time for their staff to achieve, and residents also reported being too full. This round was changed and another time slot was allocated which allowed the care home to reach $100 \%$ compliance with their seven structured drink rounds.

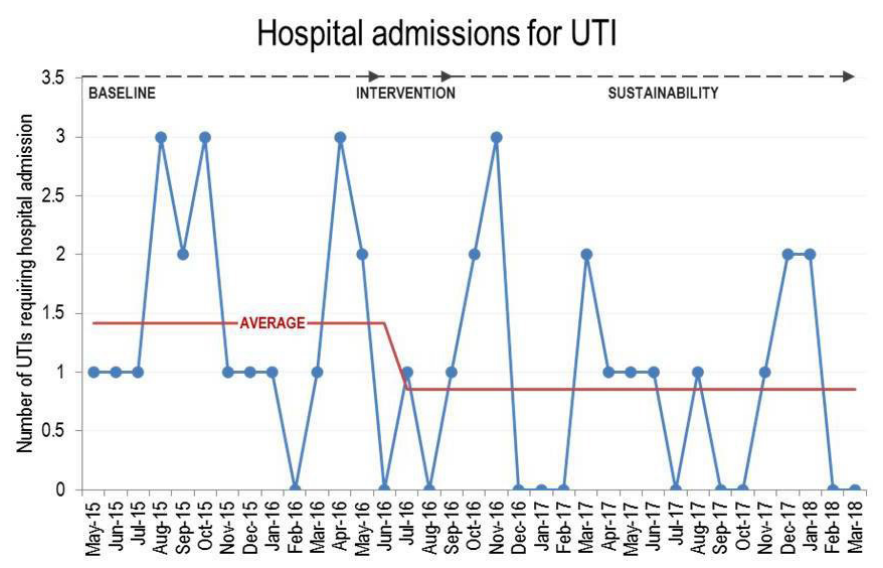

Figure 3 Average number of hospital admissions for urinary tract infections (UTIs) during the baseline, intervention and sustainability phase.
The nursing home had high staff turnover which impacted the consistency of the drink rounds. They were encouraged to re-inforce the message of hydration through safety huddles at the beginning of each shift. This worked well and staff reached $100 \%$ drink rounds compliance.

\section{Lessons and limitations}

All quality improvement programmes require review of existing research, support from leadership, engaging service users and staff. However, in this context of running a project within the care home setting, particular importance was placed on support, data and responding to a changing environment.

External support within the care home environment is limited and one of the key success factors to this project was the commitment of a team member from the East Berkshire CCG. They were trusted by the care homes and provided consistency, guidance and encouragement especially in the first 6 months of the project. It was imperative to involve staff in the design of the principle intervention to ensure that it was practical and sustainable.

Understanding data within the care home environment varies widely between staff. Having collected the baseline data, it proved invaluable for all the team to understand on average how many UTIs were occurring in their home and that they could make a difference. Moving forward, the care home managers reviewed the data with staff at regular intervals to demonstrate improvement which gave them the impetus to keep going. While data on UTI admissions to hospital and UTIs requiring antibiotics were collected, it would have been valuable to look at other projects that were occurring at the same time in the care home, for example, reduction in falls, to note if these had any influence on the outcome.

Throughout the project, UTIs were counted if the GP diagnosed it as so. In hindsight, it would have been beneficial to have clarified what constituted a confirmed diagnosis of a UTI, as practice varies between GPs.

The care home environment poses specific challenges, as at times there can be a high turnover of staff including management staff which occurred within one of the homes. Further training sessions were held, but this still did not fully address the situation. Following discussions with care home staff on what would help with training new staff, a set of small cartoon clips were designed which staff can access on YouTube. Huddles at the beginning of each shift were introduced within this care home as a means to incorporate the importance of hydration. On reflection, this might have been appropriate to introduce into each care home so that the hydration needs of all residents was a priority at the beginning of the day including residents who required particular support.

\section{CONCLUSION}

Residents of care homes are at high risk of dehydration which increases the risk of UTIs and other problems. 
This project has highlighted that a combination of optimising hydration, education for staff and clinical guidance reduces the need for antibiotics which may in turn reduce hospital admissions for UTIs. These changes have been sustained for 2 years in the four care homes engaged in the project.

Reducing admissions associated with UTIs is important because every avoided uncomplicated admission has a potential minimum saving of $£ 1331.00$ per day in the NHS. ${ }^{23}$ Reducing the use of antibiotics is cost-effective and links closely with the global ambition of antimicrobial stewardship. Care homes throughout the Thames Valley region and beyond are now adopting the principles for improvement with the aid of all the project details included in a toolkit on the Oxford Patient Safety Collaborative website. Train-the-trainer days have also been designed to support the organisations undertaking the improvement project. Six short clips incorporating the training have been designed and uploaded to YouTube which can be used by care homes to train new staff.

The success of the hydration project was down to care home staff co-designing it, so that seven structured drink rounds a day could be carried out flexibly in each care setting to marry with the existing daily routine. It is low cost to implement, easy to measure and has far surpassed the aim of reducing UTI admissions to hospital by $5 \%$ each year.

Acknowledgements We would like to thank the care home staff who co-designed, developed and implemented this intervention. Their dedication and hard work improves the lives of the older people in their care. We would like to thank the Acute Kidney Injury Workstream based at the Oxford Patient Safety Collaborative where this project was conceived.

Contributors $\mathrm{KL}$ and SJ co-designed the project, delivered the training and aggregated the data. RFN analysed the data and took the lead in writing the manuscript. CV critically reviewed the manuscript and all authors contributed to the final version.

Funding This project was not funded by a specific grant as care homes were using existing resources. Health Education England have subsequently funded video clips to support the training for this initiative (https://www.youtube.com/watch?v= oY8m3bv2JDU).

Competing interests None declared.

Patient consent for publication Consent was gained from the care home managers for the use of anonymous data. Residents involvement was part of routine daily care.

Provenance and peer review Not commissioned; externally peer reviewed.

Open access This is an open access article distributed in accordance with the Creative Commons Attribution Non Commercial (CC BY-NC 4.0) license, which permits others to distribute, remix, adapt, build upon this work non-commercially, and license their derivative works on different terms, provided the original work is properly cited, appropriate credit is given, any changes made indicated, and the use is non-commercial. See: http:// creativecommons.org/licenses/by-nc/4.0/.

\section{REFERENCES}

1. NHS England. Emergency admissions for ambulatory care sensitive conditions, 2014. Available: https://www.england.nhs.uk/wp-content/ uploads/2014/03/red-acsc-em-admissions-2.pdf [Accessed 24 Oct 2018].

2. Schols JMGA, De Groot CPGM, van der Cammen TJM, et al. Preventing and treating dehydration in the elderly during periods of illness and warm weather. J Nutr Health Aging 2009;13:150-7.

3. Courtney M, O'Reilly M, Edwards $\mathrm{H}$, et al. The relationship between clinical outcomes and quality of life for residents of aged care facilities. Aust J Adv Nurs 2009;26:49-57.

4. Hooper L, Bunn D. Detecting dehydration in older people: useful tests. Nurs Times 2015;111:12-16.

5. Campbell N. Innovations to support hydration care across health and social care. Br J Community Nurs 2016;21:S24-S29.

6. Bunn D, Jimoh F, Wilsher SH, et al. Increasing fluid intake and reducing dehydration risk in older people living in long-term care: a systematic review. J Am Med Dir Assoc 2015;16:101-13.

7. Shipman D, Hooten J. Are nursing homes adequately staffed? The silent epidemic of malnutrition and dehydration in nursing home residents. Until mandatory staffing standards are created and enforced, residents are at risk. J Gerontol Nurs 2007;33:15-18.

8. Care Quality Commission. Dignity and nutrition for older people: review of compliance. CQC, London, 2011. Available: https://www. cqc.org.uk/publications/themed-inspection/dignity-and-nutritionolder-people [Accessed 24 Oct 2018].

9. Royal College of Speech and Language Therapists. RCSLT resource manual for commissioning and planning services for SLCN: dysphagia, 2009. Available: https://www.rcslt.org/speech and_language_therapy/commissioning/dysphagia_manual_072014 [Accessed 24 Oct 2018].

10. Sura L, Madhavan A, Carnaby G, et al. Dysphagia in the elderly: management and nutritional considerations. Clin Interv Aging 2012;7:287-98.

11. Ullrich $\mathrm{S}, \mathrm{McC}$ utcheon $\mathrm{H}$. Nursing practice and oral fluid intake of older people with dementia. J Clin Nurs 2008;17:2910-9.

12. Mentes JC. The complexities of hydration issues in the elderly. Nutr Today 2013;48:S10-S12.

13. Hooper L, Abdelhamid A, Attreed NJ, et al. Clinical symptoms, signs and tests for identification of impending and current waterloss dehydration in older people. Cochrane Database Syst Rev 2015;4:1-212.

14. Mentes JCet al. Managing oral hydration. In: Boltz M, Capezuti E, Fulmer T, et al, eds. Evidence-based geriatric nursing protocols for best practice. 5th edition. New York: Springer Publishing Company, 2016: 111-24.

15. Kayser-Jones J, Schell ES, Porter C, et al. Factors contributing to dehydration in nursing homes: inadequate staffing and lack of professional supervision. J Am Geriatr Soc 1999;47:1187-94.

16. Forsyth DM, Lapid MI, Ellenbecker SM, et al. Hydration status of geriatric patients in a psychiatric hospital. Issues Ment Health Nurs 2008;29:853-62.

17. Pokrywka HS, Koffler KH, Remsburg R, et al. Accuracy of patient care staff in estimating and documenting meal intake of nursing home residents. J Am Geriatr Soc 1997;45:1223-7.

18. Jimoh FO, Bunn D, Hooper L. Assessment of a self-reported drinks diary for the estimation of drinks intake by care home residents: fluid intake study in the elderly (FISE). J Nutr Health Aging 2015;19:491-6.

19. Robinson SB, Rosher RB. Can a beverage CART help improve hydration? Geriatr Nurs 2002;23:208-11.

20. Davis C. 10 ways to reduce UTIs: long term management care. iAdv SR care. Spring 2017;66:32-4.

21. Sign.ac.uk. Management of suspected bacterial urinary tract infection in adults, 2012. Available: https://www.sign.ac.uk/sign88-management-of-suspected-bacterial-urinary-tract-infection-inadults.html [Accessed 24 Oct 2018].

22. Gleick $\mathrm{PH}$. Basic water requirements for human activities: meeting basic needs. Water Int 1996;21:83-92.

23. NHS Improvement. National tariff payment system $2017 / 18$ and 2018/19, 2016. Available: https://improvement.nhs.uk/resources/ national-tariff-1719/ [Accessed 24 Oct 2018]. 